Research Paper

\title{
A Novel Treatment Method for Lymph Node Metastasis Using a Lymphatic Drug Delivery System with Nano/Microbubbles and Ultrasound
}

\author{
Shigeki Kato1 ${ }^{1}$, Shiro Mori², Tetsuya Kodama ${ }^{\bowtie}$ \\ 1. Laboratory of Biomedical Engineering for Cancer, Graduate School of Biomedical Engineering, Tohoku University, 4-1 Seiryo, Aoba, Sendai, \\ Miyagi 980-8575, Japan \\ 2. Department of Oral Medicine and Surgery, Tohoku University Hospital, 1-1 Seiryo, Aoba, Sendai, Miyagi 980-8575, Japan. \\ $\triangle$ Corresponding author: Tetsuya Kodama, PhD., Laboratory of Biomedical Engineering for Cancer, Graduate School of Biomedical Engineering, \\ Tohoku University, 4-1 Seiryo, Aoba, Sendai, Miyagi 980-8575, Japan. Tel \& Fax: +81-22-717-7583; E-mail: kodama@bme.tohoku.ac.jp \\ ( 2015 Ivyspring International Publisher. Reproduction is permitted for personal, noncommercial use, provided that the article is in whole, unmodified, and properly cited. \\ See http://ivyspring.com/terms for terms and conditions.
}

Received: 2015.06.24; Accepted: 2015.08.31; Published: 2015.10.20

\begin{abstract}
Chemotherapy based on hematogenous administration of drugs to lymph nodes (LNs) located outside the surgically resected area shows limited tissue selectivity and inadequate response rates, resulting in poor prognosis. Here, we demonstrate proof of concept for a lymphatic drug delivery system using nano/microbubbles (NMBs) and ultrasound (US) to achieve sonoporation in LNs located outside the dissection area.

First, we demonstrated the in vitro effectiveness of doxorubicin (Dox) delivered into three different tumor cell lines by sonoporation. Sonoporation increased the Dox autofluorescence signal and resulted in a subsequent decrease in cell viability. Next, we verified the antitumor effects of Dox in vivo using MXH10/Mo-/pr/lpr mice that exhibit systemic lymphadenopathy, with some peripheral $L N s$ reaching $10 \mathrm{~mm}$ in diameter. We defined the subiliac $L N(S i L N)$ as the upstream $L N$ within the dissection area, and the proper axillary LN (PALN) as the downstream LN outside the dissection area. Dox and NMBs were injected into the SiLN and delivered to the PALN via lymphatic vessels; the PALN was then exposed to US when it had filled with solution. We found that sonoporation enhanced the intracellular uptake of Dox leading to high cytotoxicity. We also found that sonoporation induced extravasation of Dox from lymphatic endothelia and penetration of Dox into tumor tissues within the PALN. Furthermore, our method inhibited tumor growth and diminished blood vessels in the PALN while avoiding systemic toxic effects of Dox. Our findings indicate that a lymphatic drug delivery system with sonoporation represents a promising method for treating metastatic LNs located outside the dissection area.
\end{abstract}

Key words: chemotherapy; ultrasound; nano/microbubbles; cavitation; lymph node metastasis; drug delivery system

\section{Introduction}

The use of LN resection in breast or head and neck cancer is limited to patients who can tolerate surgery and whose vital organs have not been infiltrated with malignant cells [1-3]. Once metastatic cells have spread into LNs located outside the dissection area, it becomes difficult to cure a cancer completely using current medical therapies, greatly increasing the risks of mortality [4]. Thus, achieving a good outcome in cancer treatment requires appropriate control and prevention of metastasis to LNs located outside the dissection area [3]. However, no effective treatments against LN metastasis have been established to date.

Metastasis to LNs located outside the dissection area has been reported to occur in some patients only a short time after surgery [5]. Currently used diagnostic modalities such as computed tomography (CT), 
magnetic resonance imaging (MRI) and positron emission tomography (PET) are unable to detect metastatic LNs less than 7-8 $\mathrm{mm}$ in diameter. Therefore, a novel method is required to treat metastatic LNs located outside the dissection area [6].

Current chemotherapy approaches involve the injection of antitumor agents into blood vessels, and although injected drugs migrate into the interstitial spaces, they tend to be reabsorbed by capillaries rather than be taken up by lymphatic vessels (LVs) [7, 8]. Hence, it is difficult for drugs to access the lymphatic network after intravenous administration. Pioneering work by Braun et al. [9] demonstrated that a needle could be inserted directly into the afferent LV of the popliteal LN, allowing solutions to be administered into the lymphatic system. However, this procedure requires considerable skill and is difficult to achieve noninvasively. In canine and swine models, contrast agents (such as a lipid bubbles) administered subcutaneously are absorbed passively by LVs, permitting visualization of the lymphatics by diagnostic ultrasound (US) imaging [10-12]. Although subcutaneous injections are straightforward to perform, this approach is an inefficient method for drug delivery due to low tissue selectivity.

Here, we describe the novel technique of intranodal drug administration in MXH10/Mo-lpr/lpr (MXH10/Mo/lpr) mice, which exhibit LN swelling. MXH10/Mo/lpr mice show systemic lymphadenopathy, resulting in LNs whose sizes are about the same as those of human LNs [13]. Thus, these mice provide an excellent model system for the development of novel treatments for LN metastasis. In a previous study, we reported that when a mixture of fluorescent molecules and nano/microbubbles (NMBs) was injected into the SiLN, the solution was able to flow into epigastric LVs and reach the PALN [3]. Furthermore, application of ultrasound to the PALN after intranodal administration of fluorescent molecules and NMBs enhanced the delivery of fluorescent molecules into the cells of the PALN [14].

In the present study, doxorubicin (Dox) was administered to a metastatic LN using a lymphatic delivery system combined with sonoporation, and its antitumor effects were investigated. We first carried out in vitro studies to determine whether sonoporation enhanced Dox uptake into three types of tumor cell. Then, we verified whether use of the lymphatic drug delivery system in conjunction with sonoporation improved the antitumor effects of Dox in an MXH10/Mo/lpr mouse model of a tumor-bearing LN. In this model, tumor cells were inoculated into the PALN, which was defined as a metastatic LN. We defined the SiLN as the upstream LN within the dissection area, and the PALN as the downstream LN outside the dissection area.

\section{Materials and Methods}

\section{Cell lines}

KM-Luc/GFP cells, stably expressing a fusion of luciferase and green fluorescent protein genes, were prepared by transfection of malignant fibrous histiocytoma-like (MRL/N-1) cells [6] with pEGFPLuc (BD Biosciences, Franklin Lakes, NJ, USA) using lipofectin transfection reagent (Invitrogen, Carlsbad, CA, USA). MRL/N-1 cells are a sarcoma cell line established from the spleens of MRL/MpTn-gld/gld mice $[15,16]$. The LM8 murine osteosarcoma cell line and the MBT-2 murine bladder tumor cell line were purchased from the Riken BioResource Center (Tsukuba, Ibaraki, Japan).

\section{Cell culture}

KM-Luc/GFP and LM8 cells were cultivated in Dulbecco's modified Eagle's medium (DMEM; Sigma-Aldrich, St Louis, MO, USA) supplemented with $10 \%$ heat-inactivated fetal bovine serum (HyClone Laboratories Inc., South Logan, Utah, USA) and 1\% L-glutamine-penicillin-streptomycin (SigmaAldrich), whereas MBT-2 cells were cultivated in RPMI-1640 medium containing the same supplements as those added to the DMEM. KM-Luc/GFP cells were selected in $0.5 \mathrm{mg} / \mathrm{mL}$ geneticin (G418) (Wako Pure Chemical Industries, Ltd., Osaka, Japan). Cells were cultivated in a $10-\mathrm{cm}$ culture dish and maintained in a humidified incubator at $37^{\circ} \mathrm{C}$ under an atmosphere containing 5\% CO2 and 95\% air. Once confluency reached around $80 \%$, adherent cells were washed in warmed Dulbecco's phosphate buffered saline (PBS) (Sigma-Aldrich), trypsinized, and counted in a hemocytometer; dead cells were excluded using staining with trypan blue dye (Sigma-Aldrich). Before cell inoculation, we confirmed that the solutions were not contaminated by $\mathrm{Myco-}$ plasma using a Mycoplasma detection kit.

\section{Preparation of liposomes and acoustic lipo- somes}

Acoustic liposomes (AL) were used as NMBs [17]. To prepare the liposomes, 1,2-distearoyl-snglycero-3-phosphatidyl choline (DSPC; NOF, Tokyo, Japan) and 1,2-distearoyl-sn-glycero-3-phosphoethanolamine-methoxy-polyethyleneglycol (DSPE-PEG [2000-OMe]; NOF) $(94: 6 \mathrm{~mol} / \mathrm{mol})$ were placed in a pear-shaped flask, and chloroform was added until the lipids were completely dissolved. Then, the chloroform was evaporated under reduced pressure using a rotary evaporator (NVC-2100/N-1000, Eyela, Tokyo, Japan) until a lipid film remained. The flask was then placed in a vacuum desiccator overnight for 
further drying. Next, the dried film was hydrated with $5 \mathrm{~mL}$ of PBS and the resulting multilamellar vesicles subjected to 3 freeze-thaw cycles. The size of the liposomes was adjusted to $<100 \mathrm{~nm}$ using extruding equipment (Northern Lipids Inc., Vancouver, BC, Canada) with three filter sizes (100, 200 and 600 nm; Nuclepore Track-Etch Membrane, Whatman plc, Maidstone, UK) [18]. For sterilization, liposomes were passed through a $0.45 \mu \mathrm{m}$ pore size filter (Millex HV filter unit, Durapore polyvinylidine-difluoride [PVDF] membrane, EMD Millipore, Billerica, MA, USA). The lipid concentration was measured using an enzymatic method (Phospholipids C assay, Wako), according to the manufacturer's protocol. To prepare ALs, the liposomes were diluted in PBS so as to achieve a concentration of $1 \mathrm{mg} / \mathrm{mL}$ in a final volume of $1 \mathrm{~mL}$. This suspension was then sonicated with a $20-\mathrm{kHz}$ stick sonicator $(130 \mathrm{~W}$, Sonics \& Materials, Newton, CT, USA) at 50\% amplifying strength for 1 min, under a constant flow of perfluoropropane [17].

\section{Calibration and delivery of ultrasound}

The protocol for US calibration was derived from that of Watanabe et al. [19]. A 12-mm, 970-kHz, submersible US transducer (Honda Electronics, Toyohashi, Japan) was used for both in vitro and in vivo experiments. The transducer was placed in a test chamber $(600 \mathrm{~mm} \times 300 \mathrm{~mm} \times 360 \mathrm{~mm})$ filled with tap water. The $970-\mathrm{kHz}$ signals were generated by a multifunction synthesizer (WF1946A; NF Co., Yokohama, Japan) and amplified by a high-speed bipolar amplifier (HSA4101; NF Co.). The pressure values were measured using a PVDF needle hydrophone (PVDF-Z44-1100; Specialty Engineering Associates, Soquel, CA, USA) at a stand-off distance of $1 \mathrm{~mm}$ from the transducer surface, positioned using a stage control system (Mark-204-MS; Sigma Koki, Tokyo, Japan). The signals from both the amplifier and the hydrophone were recorded on a digital phosphor oscilloscope (Wave Surfer 454, $500 \mathrm{MHz}, 1 \mathrm{M} \Omega$ [16 pF]; LeCroy Co., Chestnut, NY, USA) in tap water degassed with transducer (SPN-620) generated by ultrasonic generator a2 (GP-622D) (Chiyoda Electric Co., Chikuma, Japan). For in vitro experiments, the US parameters were as follows: intensity, $0.5 \mathrm{~W} / \mathrm{cm}^{2}$ (peak negative pressure, $0.09 \mathrm{MPa}$ ); duty cycle, $50 \%$; number of pulses, 2000; pulse repetition frequency, $243 \mathrm{~Hz}$; and exposure time, $10 \mathrm{~s}$. For in vivo experiments, the corresponding parameters were: 3.0 $\mathrm{W} / \mathrm{cm}^{2}(0.21 \mathrm{MPa}) ; 20 \%$; 200; $970 \mathrm{~Hz}$; and $60 \mathrm{~s}$.

\section{In vitro study of Dox delivery into tumor cells and cell viability assays}

To study the intracellular uptake of Dox (Wako) into tumor cells, $5.0 \times 10^{3} \mathrm{KM}$-Luc/GFP cells, $5.0 \times 10^{3}$
LM8 cells, or $2.5 \times 10^{4}$ MBT-2 cells suspended in 500 $\mu \mathrm{L}$ of medium were incubated with different solutions for $24 \mathrm{~h}$ in 48-well plates; subsequently, the supernatant in each well was removed. In the control group and AL+US group, a solution containing medium $(100 \mu \mathrm{L})$ and ALs $(10 \mu \mathrm{L})$ was applied to each well. In the Dox+AL group and Dox+AL+US group, a solution containing medium $(95 \mu \mathrm{L})$, ALs $(10 \mu \mathrm{L})$ and Dox in PBS ( $5 \mu \mathrm{L} ; 0.1 \mathrm{mM}$ for KM-Luc/GFP or LM8, 0.5 $\mathrm{mM}$ for MBT-2) was applied to each well. In the $\mathrm{AL}+\mathrm{US}$ and Dox+AL+US groups, each well was positioned at a distance 1-mm from the transducer surface (which was fixed in tap water) and exposed to US. After sonication, the cells were left to incubate for $1 \mathrm{~h}$. Subsequently, Dox fluorescence in the cells was visualized by a scanning laser confocal microscope (FV300, Olympus, Tokyo, Japan) equipped with a 530-nm excitation filter, 615-nm emission filter, and $40 \times / 0.6$ dry objective lens (Olympus). To quantify the intensity of the Dox fluorescence, the red signals were converted into 8-bit values and the mean signal intensity in the region of interest (ROI; $256 \mu \mathrm{m} \times 256$ $\mu \mathrm{m})$ was calculated using ImageJ for Windows.

For cell viability assays, the cells were incubated for $1 \mathrm{~h}$ post-sonication. For KM-Luc/GFP or LM8 cells, the supernatant was removed, the cells washed twice with $300 \mu \mathrm{L}$ of PBS, and $500 \mu \mathrm{L}$ of culture medium added. For MBT-2 cells, the supernatant was removed and $500 \mu \mathrm{L}$ of culture medium was applied. After incubation for $24 \mathrm{~h}$, an MTT assay was performed, as follows: First, $250 \mu \mathrm{g}$ of 3-(4,5-di-methylthiazol-2-yl)-2,5-diphenyltetrazolium bromide (MTT; Sigma-Aldrich) solution was added to each well. After incubation for $1 \mathrm{~h}$, the supernatant was removed and $300 \mu \mathrm{L}$ of dimethyl sulfoxide (Wako) was added to each well to dissolve the formazan product. Then, $100 \mu \mathrm{L}$ of solution from each well was transferred into a 96-well plate, and the optical density read at $590 \mathrm{~nm}$ using a microplate reader (Sunrise; Tecan Japan Co., Ltd, Kawasaki, Japan). Cell viability was normalized to the value of the control group.

\section{In vivo experiments}

All animal experiments were performed in accordance with the ethical guidelines of Tohoku University. All in vivo experiments were performed under isoflurane inhalation anesthesia, and great efforts were made to minimize animal suffering.

\section{Animal model}

The MXH10/Mo/lpr mouse is a substrain of the recombinant inbred $\mathrm{MXH} / \mathrm{lpr}$ strain [13]. $\mathrm{MXH} / \mathrm{lpr}$ mice were prepared using two different parental inbred strains as progenitors, MRL/lpr and $\mathrm{C} 3 \mathrm{H} / \mathrm{lpr}$ 
mice, followed by an F1 intercross and more than 20 generations of strict brother-sister matings [14, 20]. The MXH10/Mo/lpr mouse has systemic lymphadenopathy and LNs that reach up to $10 \mathrm{~mm}$ in size after 2.5-3 months of age. Before examination, the mice were shaved and the hair around the lateral region removed by depilatory cream.

\section{Visualization of LVs and LNs}

Three female MXH10/Mo/lpr mice (20 weeks of age) were used in experiments visualizing the lymphatic routes between the SiLN and PALN. Each mouse was anesthetized with 5\% isoflurane (Abbott Japan, Tokyo, Japan) in oxygen and placed on a heated stage $\left(38^{\circ} \mathrm{C}\right)$. Then, $100 \mu \mathrm{L}$ of India ink was injected into the SiLN through a butterfly needle (27 $\mathrm{G}$, Terumo, Tokyo, Japan) attached to a 1-mL syringe (Terumo), at a rate of $50 \mu \mathrm{L} / \mathrm{min}$. Optical images of LVs and LNs were captured by a digital camera (Cannon, Tokyo, Japan). For visualization of LVs by high-frequency US imaging, 3 male MXH10/Mo/lpr mice (19 weeks of age) were anesthetized and positioned on a heated stage. The scanner was equipped with a mechanical single-element transducer (RMV-710B; central frequency, $25 \mathrm{MHz}$; axial resolution, $70 \mathrm{~mm}$; lateral resolution, $140 \mathrm{~mm}$; focal length, $15 \mathrm{~mm}$; depth of field, $2.7 \mathrm{~mm}$; VisualSonics, Toronto, ON, Canada). The transducer was fixed to a 3-D stage control system (Mark-204-MS, Sigma Koki, Tokyo, Japan). To maintain US transmission, US gel was placed on the PALN. The B-mode image was obtained as follows: The RMV-710B transducer was placed on the PALN and $150 \mu \mathrm{L}$ of ALs was injected into the SiLN at a rate of $50 \mu \mathrm{L} / \mathrm{min}$. The B-mode images were acquired from a $15.0 \mathrm{~mm} \times 15.0 \mathrm{~mm}$ area at a frame rate of $15 \mathrm{~Hz}$.

\section{Tumor implantation into the PALN}

To prepare the model of a tumor-bearing LN, 4.0 $\times 10^{5} \mathrm{KM}$-Luc/GFP cells/mL were suspended in a mixture of $13.3 \mu \mathrm{L}$ PBS and $26.7 \mu \mathrm{L}$ of $400 \mathrm{mg} / \mathrm{mL}$ Matrigel (Collaborative Biomedical Products) and injected into the PALN via a 24-gauge needle under the guidance of a high-frequency US imaging system (VisualSonics) equipped with a $25-\mathrm{MHz}$ transducer (RMV-710B). This day was defined as day 0.

\section{Dox delivery into the tumor-bearing PALN and visualization of Dox distribution in the PALN}

Twenty-seven male or female mice (16-19 weeks of age) were used in experiments carried out to visualize the distribution of Dox in the tumor-bearing PALN. Three days after cell inoculation (day 3), the mice were divided randomly into 5 groups. In the PBS alone $(n=5)$, Dox alone $(n=5)$ and Dox+AL+US $(n=$ 7) groups, solutions containing PBS, Dox in PBS and Dox with ALs (50:50 v/v), respectively, were injected into the SiLN using a 1-mL syringe (Terumo) connected to a butterfly needle ( $27 \mathrm{G}$, Terumo) at a rate of $50 \mu \mathrm{L} / \mathrm{min}$. For comparison, in the Dox alone iv group $(n=5)$ and Dox+AL+US iv group $(n=5)$, solutions containing Dox in PBS and Dox with ALs (50:50 v/v), respectively, were injected into the tail vein by bolus injection (29 G, Terumo). The Dox concentration was adjusted to $5 \mathrm{mg} / \mathrm{kg}$ body weight and the total volume was < $200 \mu \mathrm{L}$. In the Dox+AL+US and Dox+AL+US iv groups, the US transducer was placed in contact with the PALN with US transmission gel (Aquasonic 100, Parker Laboratories, Inc., Fairfield, NJ, USA) used as a coupling medium. US waves (970 $\mathrm{kHz}$ ) were generated and amplified using the settings described above. Half an hour after treatment, each mouse was deeply anesthetized, cervical dislocation performed, and the PALN extracted and embedded in optimal cutting temperature compound (Sakura Fineteck Japan, Tokyo, Japan). Subsequently, samples were frozen in liquid nitrogen and sectioned $(8-10 \mu \mathrm{m}$ thickness) with a cryostat (Thermo Fisher Scientific, Barrington, IL, USA). Nuclei were stained with 100 ng/mL 4',6-diamidino-2-phenylindole (DAPI; Sigma-Aldrich) at room temperature. For the experimental groups receiving intranodal administration of agents, LVs were detected using rabbit anti-lymphatic vessel endothelial hyaluronan receptor-1 (LYVE-1) primary antibody (ReliaTech $\mathrm{GmbH}$, Wolfenbüttel, Germany) and Alexa-488-conjugated goat anti-rabbit secondary antibody (Invitrogen). For the experimental groups receiving intravenous administration of agents, blood vessels were detected using rat anti-CD31 primary antibody (BD Biosciences) and Alexa-488-conjugated goat anti-rat secondary antibody (Invitrogen). Sections were then mounted under coverslips using Vectashield mounting medium (Vector Laboratories, Burlingame, CA, USA) and immunofluorescence examined using a fluorescence microscope (BX51, Olympus) equipped with a WU filter set (emission, 330-385 nm; excitation, $420 \mathrm{~nm}$ ) for DAPI, a U-NIBA filter set (emission, $470-495 \mathrm{~nm}$; excitation, $510-550 \mathrm{~nm}$ ) for Alexa-488, and a WIG filter set (emission, 530-550 nm; excitation, $575 \mathrm{~nm}$ ) for Dox. To visualize the distribution of Dox in the PALN, the Dox fluorescence signal was converted into 8-bit values and the mean fluorescence intensity calculated using ImageJ.

\section{Treatment of the tumor-bearing PALN by in- tranodal administration of Dox and sono- poration}

To verify the antitumor effects of Dox after its 
intranodal administration in combination with sonoporation, 29 male or female mice (16-20 weeks of age) were used. Mice were divided randomly into 6 groups. In the PBS alone group $(n=5), \mathrm{PBS}(<200 \mu \mathrm{L})$ was injected into the SiLN. In the Dox alone $(n=5)$ and Dox+US $(n=5)$ groups, Dox in PBS was injected into the SiLN. In the Dox+AL+US group $(n=5)$, Dox and ALs $(50: 50 \mathrm{v} / \mathrm{v})$ were injected into the SiLN. In these intranodal administration groups, the solutions were injected at a constant rate as described above. For comparison, in the Dox alone iv $(n=5)$ and Dox+AL+US iv $(n=4)$ groups, Dox in PBS and Dox with ALs (50:50 v/v), respectively, were injected into the tail vein by bolus injection. The Dox concentration was adjusted to $5 \mathrm{mg} / \mathrm{kg}$ body weight and the total administration volume was $<200 \mu \mathrm{L}$. In the Dox+US, Dox+AL+US and Dox+AL+US iv groups, the US transducer was placed in contact with the PALN and $970-\mathrm{kHz}$ US was applied to the PALN using the method described above. These therapeutic interventions were performed on day 1 and day 3, post-cell inoculation. To evaluate the tumor growth rate in the PALN, luciferase activity was measured on days 1,3 , 4, 7 and 10, post-cell inoculation. Luciferin (150 $\mathrm{mg} / \mathrm{kg}$, Promega) was injected intraperitoneally using a 1-mL syringe with a $27 \mathrm{G}$ needle (Nipro, Osaka, Japan). After $10 \mathrm{~min}$, luciferase bioluminescence was measured for $30 \mathrm{sec}$ using an in vivo luminescence imaging system (IVIS; Xenogen, Alameda, CA, USA).

\section{Immunohistochemical analysis of the PALN}

After in vivo luminescence imaging had been performed on day 10, the mouse was anesthetized deeply and cervical dislocation was performed. The PALN was excised, fixed overnight in $10 \%$ formalin solution (Wako) at room temperature, dehydrated, and then embedded in paraffin. The embedded specimens were cut into 3-4 $\mu \mathrm{m}$ serial sections and immunostained for detection of CD31-positive cells using a Discovery XT automated staining processor (Ventana Medical Systems, Inc., Tucson, AZ, USA). Immunostaining of vascular endothelial cells was achieved by incubation with a prediluted polyclonal rabbit anti-CD31 antibody (1/100 dilution; Santa Cruz Biotechnology, Inc., Santa Cruz, CA, USA) for $2 \mathrm{~h}$ at room temperature, followed by application of an anti-rabbit IgG Histofine MAX-PO (R) kit for $16 \mathrm{~min}$ at room temperature. This procedure was performed using the method described by $\mathrm{Li}$ et al. [6]. The hotspot method was used for calculating the vascular ratio [21]. Four hot-spot fields with the highest vessel density were selected under low magnification $(\times 40)$, using an optical microscope connected to a digital camera (DP72; Olympus), in the boundary region between the tumor and non-tumor tissue, and in a peripheral region some distance from the tumor region. To calculate the vascular ratio, CD31-stained vessels were traced manually, the area occupied by each vessel calculated from the number of positively-immunostained pixels, and the summed value divided by the total area of the specimen $(820 \mu \mathrm{m} \times 620$ $\mu \mathrm{m})$ [16], using ImageJ.

\section{Evaluation of local or systemic cytotoxicity of Dox}

To evaluate any acute toxic effects of Dox, the body weights of the mice were measured and recorded. In the images of the PALN, linear measurements were made across each axis to determine the length, width and depth of the PALN. The center of the tumor was set at the focal length. 2D/3D images of the tumor were constructed from B-mode images. 2D B-mode images at the center of the longitudinal length were acquired. Consecutive B-mode images, with a slice thickness of $100 \mu \mathrm{m}$, were acquired by scanning across the tumor with the aid of a computer-controlled 3D motorized stage; these were reconstructed into a 3D image, and the volume (consisting of multiple polygons) was calculated using Vevo 770 software [21].

\section{Statistical analysis}

All data are expressed as the mean \pm standard deviation (SD), or mean \pm standard error of the mean (SEM). Statistical analysis was performed by one-way ANOVA, followed by a Tukey-Kramer multiple comparison test. If data were not normally distributed, Kruskal-Wallis and Steel tests were used. $P<0.05$ was regarded as a statistically significant difference.

\section{Results}

\section{In vitro study of Dox delivery into tumor cells using ALs and US}

We hypothesized that ALs would enhance the intracellular uptake of Dox by tumor cells, and that synergistic effects in the presence of US would lead to cell death. To optimize the delivery of Dox into tumor cells by ALs and concomitant US, we investigated the delivery efficiency in vitro. Figure 1A shows confocal images of KM-Luc/GFP cells $1 \mathrm{~h}$ after sonication. In the control and AL+US groups, Dox fluorescence was not detected. Although Dox fluorescence was readily detected in the Dox+AL group, it was observed with much stronger intensity in the Dox+AL+US group, where it was clearly localized to the nuclei. To quantify the Dox fluorescence signals, the fluorescence intensity was calculated in an arbitrary ROI ( $256 \mu \mathrm{m} \times$ $256 \mu \mathrm{m})$ in each well. Figure 1B shows the mean Dox fluorescence intensity in KM-Luc/GFP cells. The mean signal intensity was significantly higher in the 
Dox + AL group $(P<0.05)$ and Dox + AL + US group $(P<$ $0.01)$ than in the control and AL+US groups. Furthermore, mean Dox fluorescence intensity was higher in the Dox+AL+US group than in the Dox+AL group $(P<0.01)$. Similar experiments were conducted in LM8 and MBT-2 cells. Additional File 1: Supplementary Figure S1A and B show confocal microscopy images of LM8 cells and MBT-2 cells, respectively, $1 \mathrm{~h}$ after sonication. As was the case for KM-Luc/GFP cells, Dox fluorescence was detected in the Dox+AL groups in both LM8 and MBT-2 cells, and US exposure (Dox+AL+US groups) enhanced Dox fluorescence in both cell lines. Figure $1 \mathrm{C}$ shows measure-

(A)
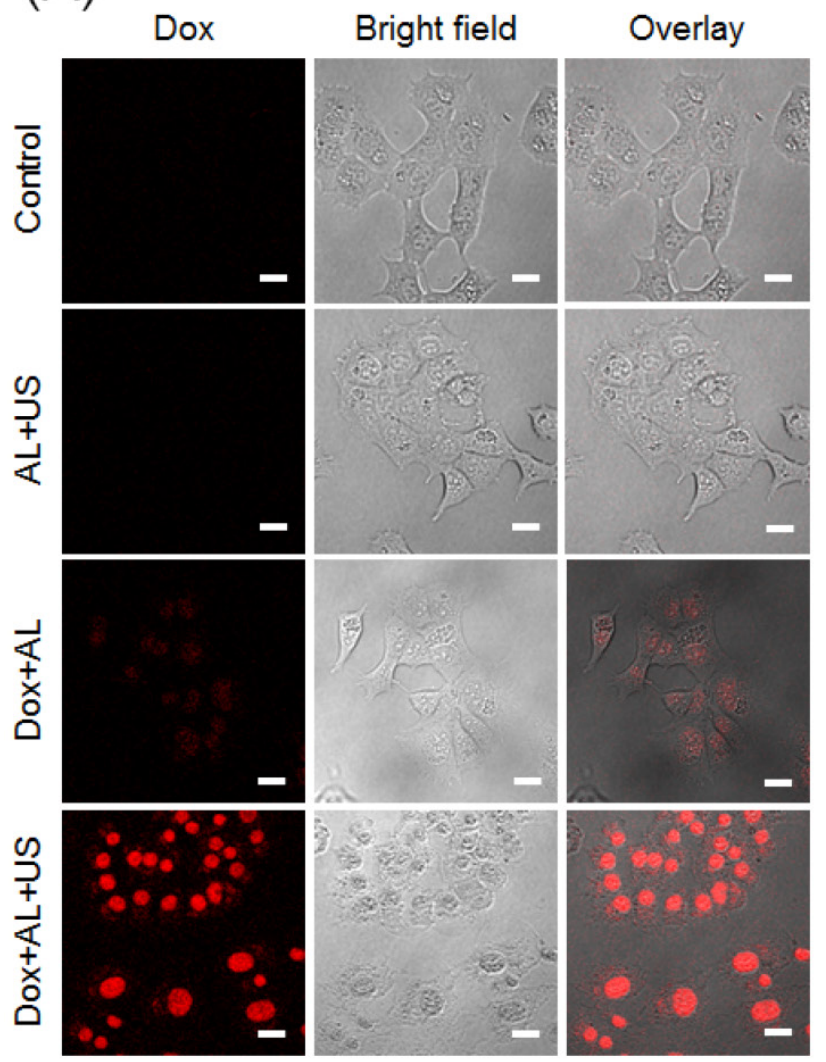

ments of the mean Dox fluorescence intensity in LM8 cells. Although the signal intensity was numerically higher in the Dox+AL group than in the control or AL+US groups, statistical significance was not reached. However, mean Dox fluorescence was significantly higher in the Dox+AL+US group than in the other 3 groups $(P<0.01)$. Figure 1D shows corresponding data in MBT-2 cells. Mean Dox fluorescence intensity was significantly higher in the Dox $+\mathrm{AL}$ group than in the control and AL+US groups $(P<$ 0.01 ), and was significantly higher in the Dox+AL+US group than in the Dox+AL $(P<0.05)$, AL+US $(P<$ $0.01)$ and control $(P<0.01)$ groups.
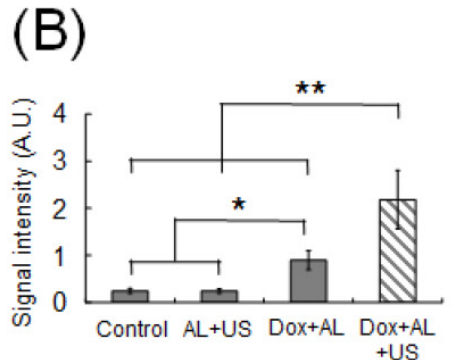

\section{(C)}
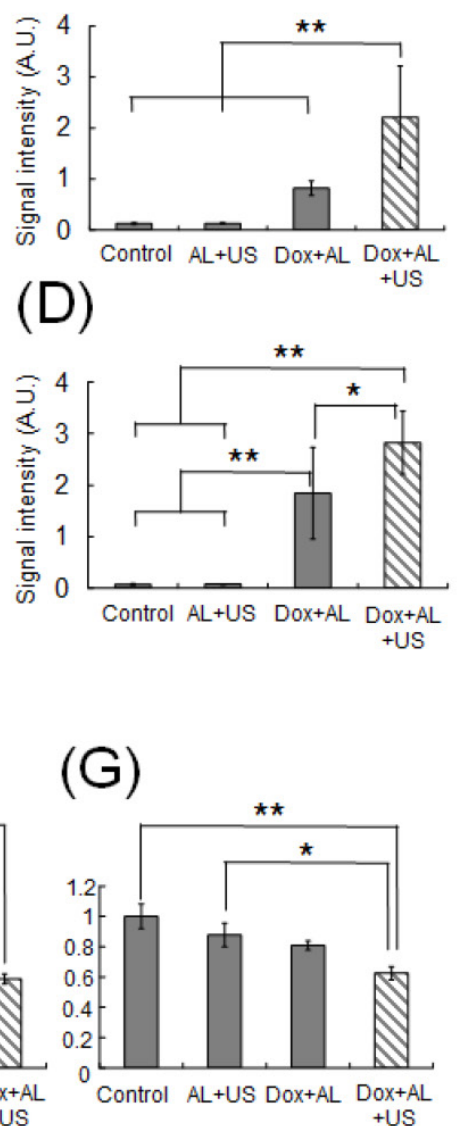

Figure 1. In vitro study of Dox internalization into tumor cells using ALs in the presence of US and evaluation of cell viability. (A) Representative confocal images of KM-Luc/GFP cells $1 \mathrm{~h}$ after sonication. Scale bars represent $20 \mu \mathrm{m}$. (B) Mean Dox fluorescence intensity in KM-Luc/GFP cells $1 \mathrm{~h}$ after sonication. Control $(n=5)$, AL+US $(n=5)$, Dox $+A L$ $(n=5)$, Dox $+A L+U S(n=5)$. (C) Mean Dox fluorescence intensity in LM8 cells 1 h after sonication. Control $(n=5), A L+U S(n=5), D o x+A L(n=5), D o x+A L+U S(n=5)$. $(D)$ Mean Dox fluorescence intensity in MBT-2 cells $1 \mathrm{~h}$ after sonication. Control $(n=5)$, AL+US $(n=5)$, Dox + AL $(n=5)$, Dox $+A L+U S(n=5)$. $(E)$ Normalized KM-Luc/GFP cell viability. Control $(n=5), A L+\cup S(n=5)$, Dox $+A L(n=5)$, Dox $+A L+U S(n=5)$. (F) Normalized LM8 cell viability. Control $(n=5), A L+U S(n=5), \operatorname{Dox}+\operatorname{AL}(n=5), \operatorname{Dox}+\operatorname{AL}+U S$ $(n=5)$. (G) Normalized MBT-2 cell viability. Control $(n=5)$, AL+US $(n=5)$, Dox $+A L(n=5)$, Dox $+A L+U S(n=5)$. Error bars represent the SD. Statistical comparisons were made using one-way ANOVA with a Tukey-Kramer post-hoc test. $* P<0.05, * * P<0.01$. 
To evaluate cell viability, an MTT assay was performed. Figure 1E shows KM-Luc/GFP cell viability in each experimental group, with each value normalized to that of the control. Cell viability did not differ significantly between the control, AL+US and Dox+AL groups. In contrast, cell viability was significantly reduced in the Dox+AL+US group compared with the control $(P<0.01)$, AL+US $(P<0.01)$ and Dox + AL $(P<0.05)$ groups. Figure $1 F$ presents data for LM8 cell viability. Cell viability was significantly decreased in the AL+US and Dox+AL groups compared with the control group $(P<0.05$ and $P<0.01$, respectively). Cell viability in the Dox+AL+US group was significantly lower than that in the control or AL+US groups $(P<0.01)$, and was numerically lower than that in the Dox+AL group, although statistical significance was not attained. Figure 1G shows MBT-2 cell viability under each experimental condition. Although cell viability in the AL+US and Dox $+\mathrm{AL}$ groups appeared to be lower than that in the control group, statistical significance was not reached. Cell viability in the Dox+AL+US group was significantly decreased compared to the control $(P<0.01)$ and AL+US $(P<0.05)$ groups, but was not significantly different from that in the Dox+AL group.

\section{Visualization of LVs and LNs in MXH10/Mo/lpr mice}

The concept of intranodal administration in this study involved the delivery of drugs to downstream LNs via the LVs. Therefore, it was important to verify in our animal model whether a drug could reach a downstream LN (PALN) via LVs after its administration in an upstream LN (SiLN) (Fig. 2A). Figure 2B shows the visualized lymphatic routes from the SiLN to the PALN, revealed by injecting India ink into the SiLN. India ink reached the PALN (Fig. 2C) after flowing through the efferent LVs (Fig. 2D) of the SiLN (Fig. 2E).

Figure $2 \mathrm{~F}$ shows a B-mode US image before injection of ALs. After the ALs had flowed through LVs to reach the PALN, an increase in brightness was detected in the PALN (Fig. 2G). According to the analysis of temporal sequence data (Additional File 2: supplementary video SV1), ALs from afferent LVs entered the marginal sinus and then flowed into the lymphatic sinus.

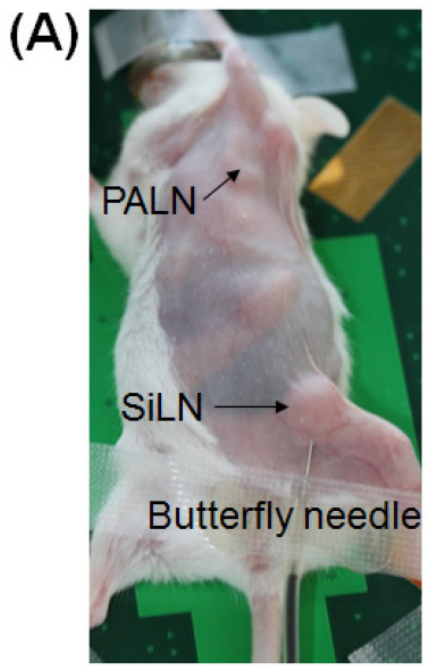

(B)

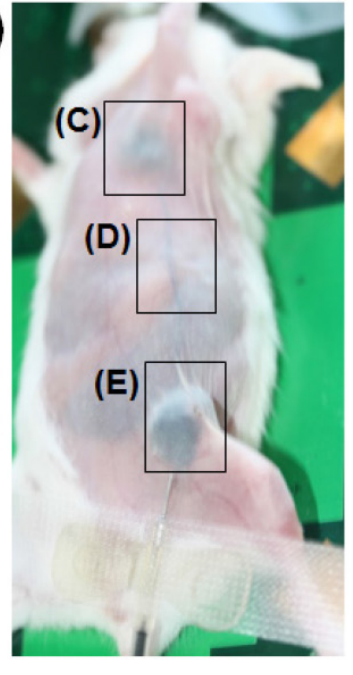

(C)

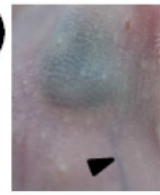

(D)

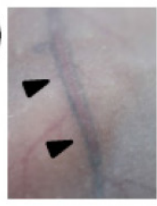

(E)

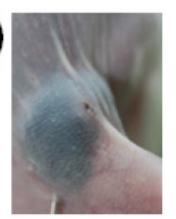

(F)

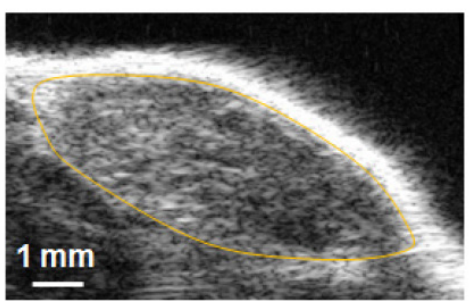

(G)

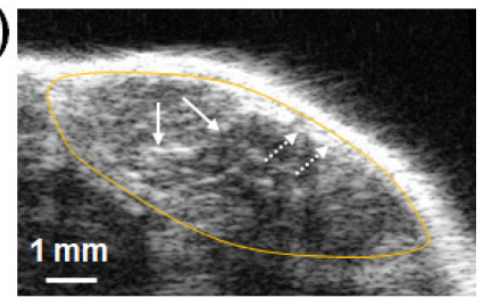

Figure 2. Visualization of the SiLN, PALN and connecting LVs in the MXH10/Mo/lpr mouse. (A) A MXH10/Mo/lpr mouse under inhalation isoflurane anesthesia. The injection of India ink was made through a $27 \mathrm{G}$ butterfly needle inserted into the SiLN and fixed by surgical tape. (B) The MXH10/Mo/lpr mouse shown in (A) immediately after injection of $100 \mu \mathrm{L}$ of India ink into the SiLN. The India ink reached the PALN (C) via epigastric LVs (D) after its injection into the SiLN (E). Rectangles show epigastric LVs stained by India ink. (F) B-mode US image of the PALN before injection of ALs. (G) B-mode US image of the PALN immediately after the injection of $150 \mu L$ of ALs into the SiLN in the same manner as that for India ink. The ALs entered the marginal sinus (dotted arrows) from afferent LVs, and then flowed into the lymphatic sinus (solid arrows). The yellow oval represents the outline of the PALN. 
(A)

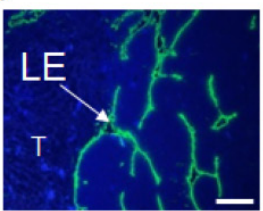

PBS alone

(B)

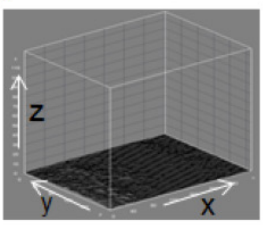

PBS alone

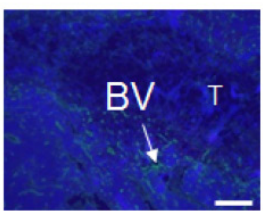

Dox alone iv

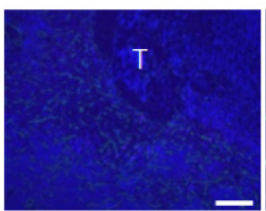

Dox+AL+US iv

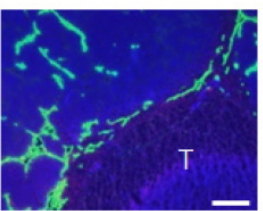

Dox alone

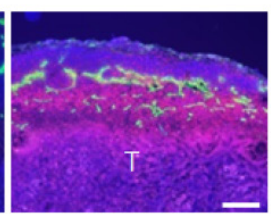

Dox+AL+US

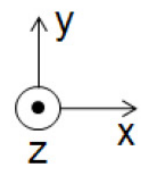

z

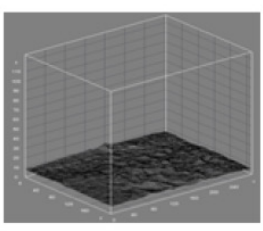

Dox alone iv

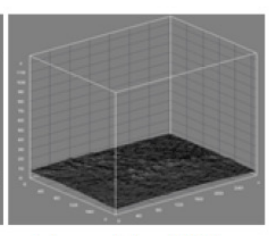

Dox+AL+US iv

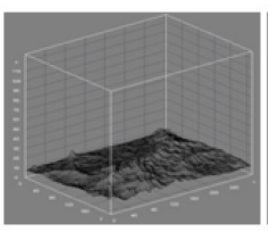

Dox alone

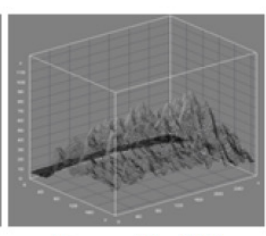

Dox+AL+US

(C)

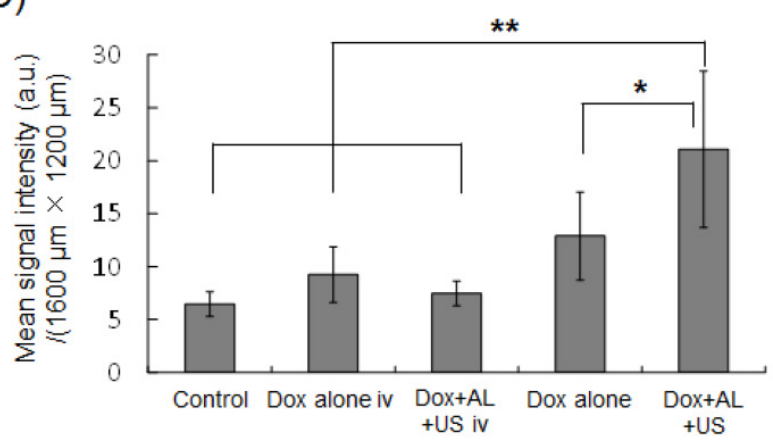

Figure 3. Delivery of Dox into the tumor-bearing PALN using intranodal administration with ALs in the presence of US. (A) Representative immunofluorescence images of tumor-bearing PALNs half an hour after treatment. The nuclei were stained blue with DAPI; the lymphatic endothelia (LE) or blood vessels (BV) were stained green with Alexa-488-conjugated anti-LYVE-1 antibody or anti-CD31 antibody; and the red regions indicate the areas to which Dox was delivered. Little or no Dox fluorescence was detected in the PBS alone $(n=5)$, Dox alone iv $(n=5)$ and Dox+AL+US iv $(n=5)$ groups. Weak Dox fluorescence was detected around the LE in the Dox alone (i.e. intranodal administration) group $(n=5)$. Dox fluorescence of strong intensity was detected around the LE in the Dox $+A L+U S$ group $(n=7)$, and Dox reached the tumor tissues within the PALN (T: tumor). Scale bars represent $200 \mu \mathrm{m}$. (B) Distribution of the gray-scale intensity of the red signals shown in Figure $3 \mathrm{~A}$. Exposure to ALs and US substantially enhanced the delivery of Dox into the tumor-bearing PALN. (C) Mean gray-scale intensities of the Dox red signals in the ROls (1600 $\mu \mathrm{m} \times 1200 \mu \mathrm{m})$. Error bars represent the SD. Statistical comparisons were made using one-way ANOVA and a Tukey-Kramer post-hoc test. $* P<0.05, * * P<0.01$.

\section{In vivo study of Dox delivery into the tu- mor-bearing PALN using intranodal admin- istration with concomitant US}

Our in vitro study demonstrated that ALs in the presence of US could enhance the delivery of Dox into tumor cells and augment the antitumor effects of Dox, raising the possibility that similar effects might be achieved in vivo. To test this, Dox was co-administered with ALs into LVs via the SiLN, and the tumor-bearing PALN was subsequently exposed to US. Figure $3 \mathrm{~A}$ shows representative immunofluorescence staining of the PALN. Nuclei are stained blue (DAPI), lymphatic endothelia or blood vessels are stained green (Alexa-488), and Dox fluorescence is visible as red. The letter ' $\mathrm{T}$ ' delineates the region of the PALN containing tumor. In the intravenous administration groups (Dox alone iv or Dox+AL+US iv), only limited Dox fluorescence was detected around the blood vessels. Following intranodal administration, Dox fluorescence was detected parallel to the lymphatic endothelia or marginal sinus. In the Dox+AL+US (intranodal administration) group, strong Dox fluorescence was detected not only around the marginal sinus but also in the medulla, where lymphocytes or tumor tissue were located. Figure 3B shows the distribution of Dox fluorescence intensity in each Dox image shown in Figure 3A. Red signals were not detected in animals that received PBS alone or intravenous administration of agents (Dox alone iv and Dox+AL+US iv groups). However, increased signals were detected around the marginal sinus in animals given intranodal Dox (Dox alone group), and even stronger signals were evident in the Dox+AL+US group. Figure 3C illustrates the mean integrated value of the red signal (in gray scale) under each set of experimental conditions. The mean value measured in the Dox+AL+US (intranodal administration) group was significantly greater than that in the control (PBS alone; $P<0.01)$, Dox alone iv $(P<0.01)$, Dox $+\mathrm{AL}+\mathrm{US}$ iv $(P<0.01)$ or Dox alone (intranodal administration; $P<0.05$ ) group, while there were no significant differences between the Dox alone (intranodal administration) group and the Control, Dox alone iv or Dox $+\mathrm{AL}+\mathrm{US}$ iv groups. 
(A)

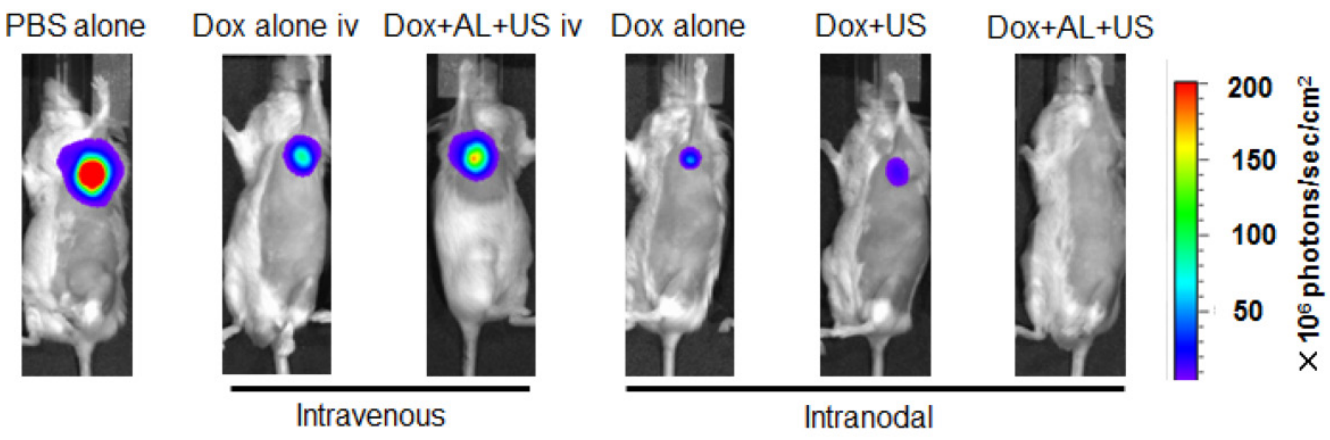

(B)

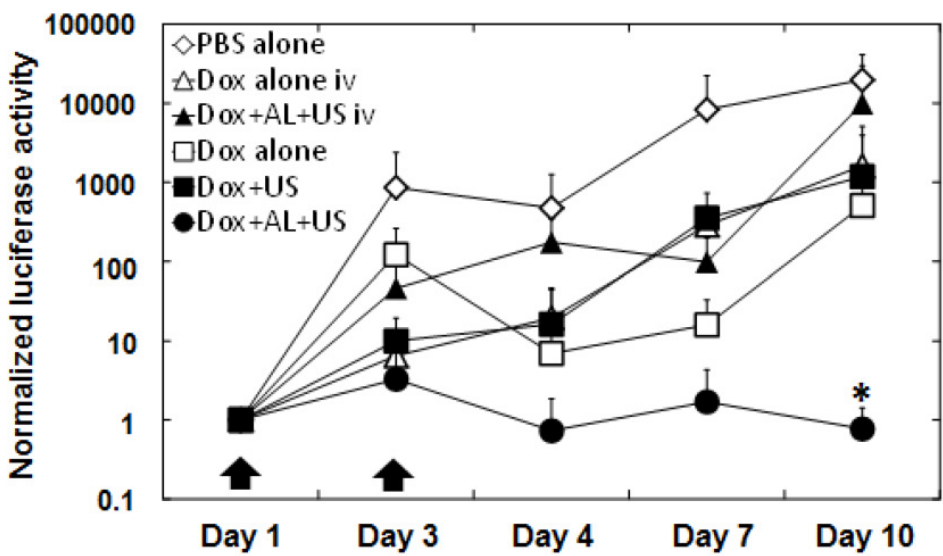

Figure 4. Quantification of temporal changes in luciferase activity in the tumor-bearing PALN. (A) Representative bioluminescence images showing luciferase activity on day 10. In the PBS alone group, strong bioluminescence was observed indicating the presence of tumor cells. In the Dox alone iv, Dox+AL+US iv, Dox alone and Dox+US groups, relatively strong bioluminescence was also observed on day 10 . Very little bioluminescence was observed in the Dox $+A L+U S$ group. (B) Normalized luciferase activity in the tumor-bearing PALN. Values at different time points were normalized to those obtained on day 1 . Black arrows indicate the day on which each treatment was administered (days 1 and 3). PBS alone $(n=5)$, Dox alone iv $(n=5)$, Dox $+A L+U S$ iv $(n=4)$, Dox alone $(n=5)$, Dox $+U S(n=5)$, Dox $+A L+U S(n=5)$. Error bars represent the SD. Statistical comparisons were made using the Kruskal-Wallis and Steel tests. $* P<0.05$.

\section{Antitumor effects of intranodal administration of Dox and ALs with concomitant US}

Having shown that the intranodal administration of Dox and ALs with concomitant US exposure enhanced Dox extravasation into the medulla where tumor tissue resided, we investigated the antitumor effects of intranodal administration of Dox and ALs combined with US exposure, using bioluminescence imaging of the tumor-bearing PALN. Figure 4A shows a series of representative bioluminescence images on day 10. When intranodal administration of Dox and ALs, with concomitant exposure to US, was performed on days 1 and 3, normalized luciferase activity was significantly lower than that in the control (PBS alone) group $(P<0.05$; Fig. 4B). There was also a trend for the normalized luciferase activity on day 10 to be lower in the Dox+AL+US group than in the Dox alone iv, Dox+AL+US iv, Dox alone and Dox+US groups, although the differences were not statistically significant.

\section{Histopathological analysis}

Histopathological analysis was performed to determine whether structural changes occurred in the tumor-bearing PALN after treatment. Figure 5A shows representative sections stained with anti-CD31 antibody, taken from an area of the PALN in which tumor tissue bordered non-tumor tissue. In this region of the PALN, the blood vessel lumens were smaller in the Dox alone and Dox+AL+US groups than in the PBS alone and Dox alone iv groups. Figure $5 \mathrm{~B}$ shows representative images of CD31 immunostaining in peripheral regions of the PALN distant to the tumor-containing area. No structural changes in these regions were observed in any of the groups. Figure 5C illustrates the CD31-positive blood vessel density in the border area between tumor and non-tumor tissue. Blood vessel density was significantly lower in the Dox alone iv and Dox alone groups than in the control group $(P<0.05)$, and was also sig- 
nificantly lower in the Dox+AL+US group than in the control group $(P<0.01)$. Figure 5D illustrates CD31-positive blood vessel density in peripheral regions distant from the tumor site: in these areas, there were no statistically significant differences between the 4 groups.

\section{Evaluation of local or systemic cytotoxicities of the treatments}

To evaluate potential local tissue toxicity of each treatment, the volume of the tumor-bearing PALN was measured using 3D high-frequency US imaging. Figure 6A shows representative 3D-reconstructed images of tumor-bearing PALNs on days 2, 5 and 9. Morphological changes were not observed in the PBS alone (control) group. However, the volume of the PALN decreased over time in the Dox alone iv, Dox alone and Dox $+\mathrm{AL}+\mathrm{US}$ groups. Figure $6 \mathrm{~B}$ illustrates the temporal changes in volume normalized to the values obtained on day 2. In the Dox alone iv and Dox alone groups, the normalized volume appeared to be lower than that in the PBS alone group on days 5 and 9 , but statistical significance was not reached. The normalized volume of the tumor-bearing PALN in the Dox+AL+US group was significantly lower than that in the PBS alone group on day $5(P<0.05)$ and day 9 $(P<0.01)$. Next, body weight changes were analyzed to determine whether the treatments were associated with any systemic toxic effects. Figure 6C illustrates the temporal changes in body weight normalized to the values obtained on day 0 before cell inoculation. There were no statistically significant differences between the PBS alone and Dox+AL+US groups. However, body weight was significantly lower in the Dox alone iv group than in the PBS alone group on days 7 and $10(P<0.01)$.

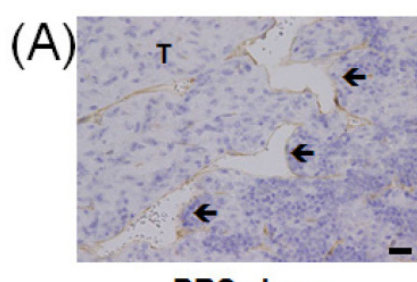

PBS alone

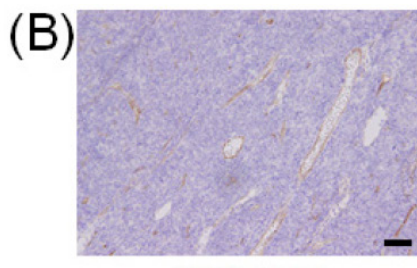

PBS alone

(C)

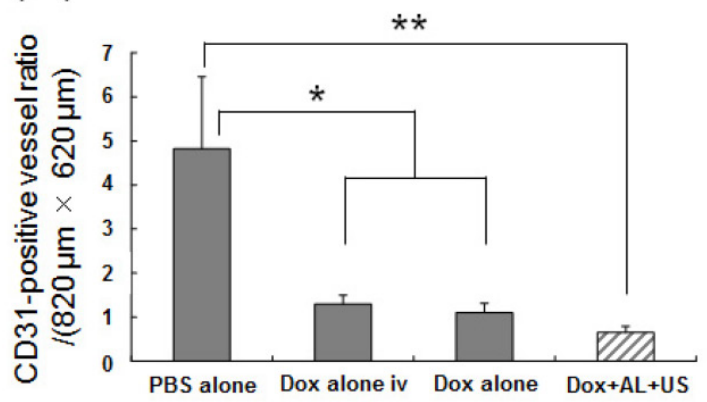

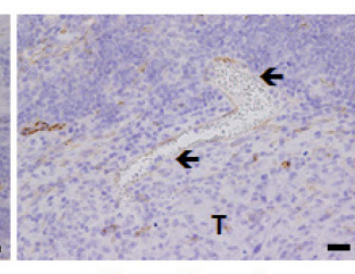

Dox alone iv

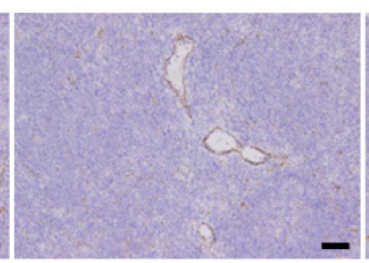

Doxalone iv

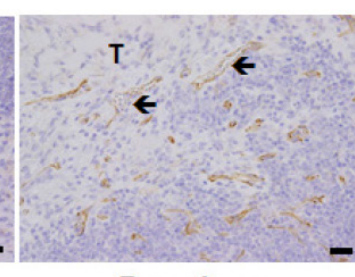

Doxalone

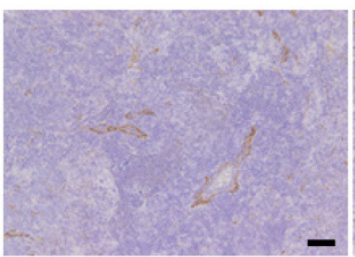

Dox alone

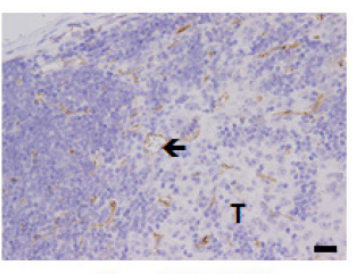

Dox+AL+US

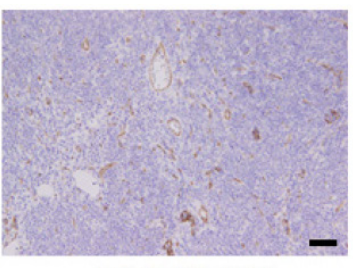

Dox+AL+US

(D)

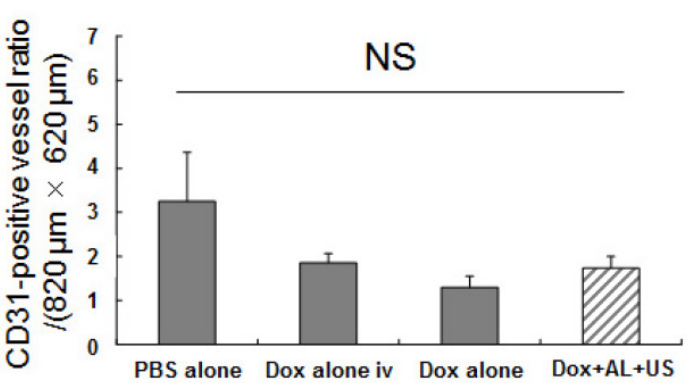

Figure 5. Evaluation of antitumor effects based on histological analysis of the PALN. (A) Representative images showing immunostaining with anti-CD 31 antibody in the region of the PALN containing the border between tumor and non-tumor tissue. The blood vessel lumens (arrows) were smaller in the Dox alone and Dox+AL+US groups than in the PBS alone and Dox alone iv groups. Scale bars represent $20 \mu \mathrm{m}$. (B) Representative images showing immunostaining with anti-CD31 antibody in a peripheral region of the PALN distant from the tumor tissue. There were no differences in blood vessel structure between any of the groups. Scale bars represent $50 \mu \mathrm{m}$. (C) CD31-positive blood vessel density in the region of the tumor-bearing PALN containing the border between tumor and non-tumor tissue. (D) CD31-positive blood vessel density in a peripheral region of the tumor-bearing PALN distant from the tumor. PBS alone $(n=4)$, Dox alone iv $(n=4)$, Dox alone $(n=4)$, Dox + AL+US $(n=4)$. Error bars represent the SEM. Statistical comparisons were made using one-way ANOVA with a Tukey-Kramer post-hoc test. $* P<0.05$, ** $P<0.01$; NS: not significant. 
(A)

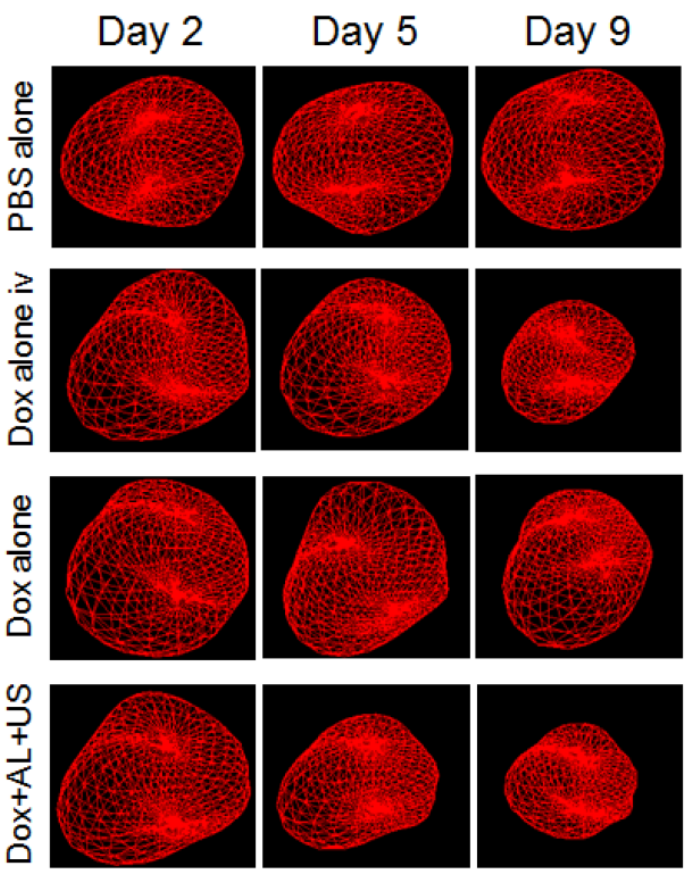

(B)

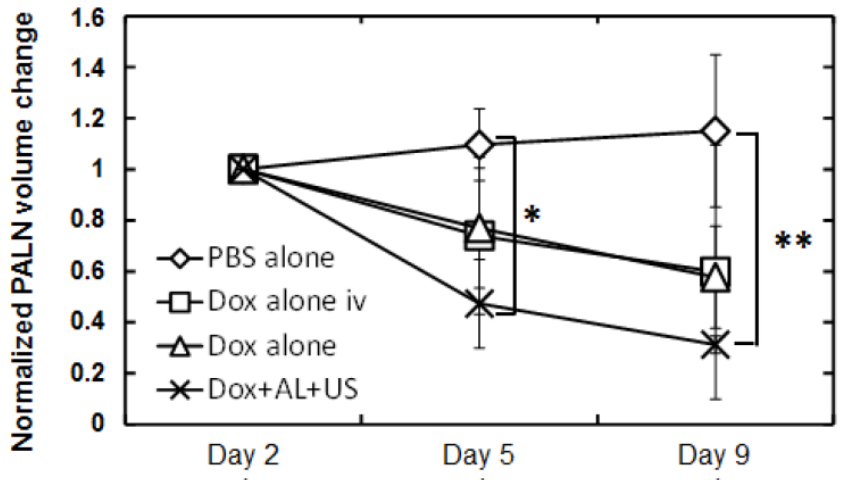

(C)

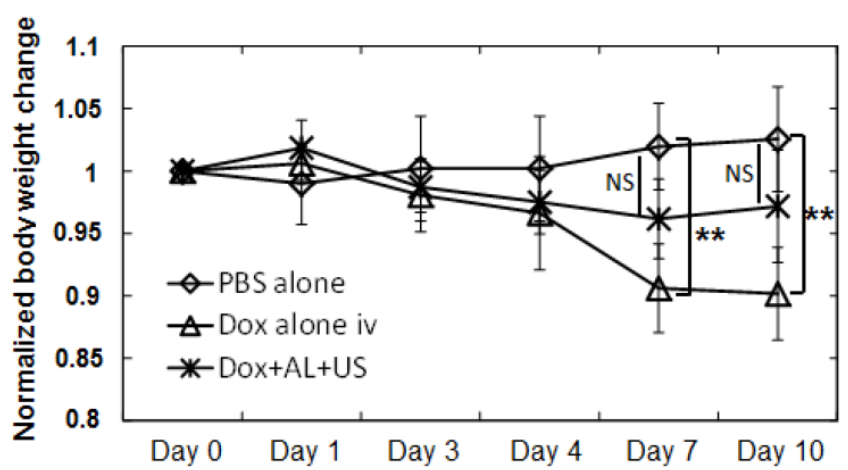

Figure 6. Evaluation of local or systemic toxicity of Dox. (A) Morphological changes in tumor-bearing PALNs observed by high-frequency US imaging. A 3D image was acquired as follows: high-frequency (central frequency, $25 \mathrm{MHz}$; axial resolution, $70 \mu \mathrm{m}$ ) B-mode US images were captured across the entire tumor-bearing PALN in $100 \mu \mathrm{m}$ steps, and the B-mode images were integrated and converted into a 3D image by VEVO software. (B) Temporal changes in the tumor-bearing PALN volume normalized to the value obtained on day 2. PBS alone $(n=5)$, Dox alone iv $(n=5)$, Dox alone $(n=5)$, Dox $+A L+U S(n=5)$. (C) Temporal changes in mouse body weight normalized to the value obtained on day 0 before cell inoculation. PBS alone $(n=5)$, Dox alone iv $(n=5)$, Dox $+A L+U S(n=5)$. Error bars represent the SD. Statistical comparisons were made using one-way ANOVA and a Tukey-Kramer post-hoc test. $* P<0.05$, $* * P<0.01$.

\section{Discussion}

This is the first paper to demonstrate that a drug delivered through the lymphatic network was able to exert a therapeutic effect in a tumor-bearing $\mathrm{LN}$, and a high antitumor activity was achieved when sonoporation was also used. When a solution of Dox and NMBs was injected into the SiLN, it successfully reached the PALN (i.e., downstream $\mathrm{LN}$ ) via the epigastric LVs, even if a portion of the injected solution infiltrated the blood vessels in the $\operatorname{LN}[3,22,23]$. Dox was able to extravasate from the lymphatic channels and penetrate into the tumor in the PALN, and exposure of the PALN to US resulted in an effective antitumor action. Furthermore, compared with the intravenous route, the use of lymphatic Dox administration with sonoporation reduced the occurrence of acute toxicity.

The mechanism underlying sonoporation most likely involves impulsive pressures exerted by the collapse of NMBs and subsequent cavitation bubbles [14], resulting in transient membrane permeability
[24, 25]. In a preclinical model of a solid tumor utilizing hematogenous administration of agents, cavitation was found to disrupt the junctions between endothelial cells, causing drug extravasation from the blood vessels [26, 27].

In present study, the methodology was applied to the treatment of $\mathrm{LN}$ metastasis in a model of a metastatic LN located outside the surgical dissection area. The LN-mediated drug administration technique used in our study has the potential to deliver drugs to metastatic LNs via the metastatic routes.

In recent years, neo-adjuvant chemotherapy with molecular target drug exerted a curative influence on many solid tumors including breast cancer in clinic $[28,29]$. For early stage of breast cancer patients, monitoring of primary tumor to drug response by conventional clinical US imaging and MRI has contributed to develop a proper treatment strategy and improved treatment outcomes [30,31]. Similarly, it is important to develop a method to monitor metastatic LNs in neo-adjuvant chemotherapy for later stages of patients. As shown in the present results, 
LN-mediated administration of NMBs combined with US imaging have the possibility of identifying a tumor-draining LNs precisely. Furthermore, monitoring of three-dimensional morphological changes by US imaging is expecting to evaluate the drug response promptly and decide the treatment strategy properly, which improve patients' quality of life and treatment outcome.

However, there are several limitations to our study. First, we regarded the tumor allograft in the PALN as a quasi-LN metastasis model. Metastatic cells in LVs become arrested around the junction of the afferent lymph duct [32], and metastatic foci are believed to form around the peripheral region of a $\mathrm{LN}$. The distribution of the foci of our tumor allograft in the PALN differs from the actual pattern of lymphatic metastasis. If the administration routes overlap with metastatic routes, metastatic foci may block the lymphatic route. In addition, if metastatic foci form outside the area exposed to US, cavitation may not affect drug delivery into metastatic cells. Second, the correlation between intralymphatic tumor volume and bioluminescence intensity remains unclear. Though it has been reported that tumor volume in a subcutaneous solid tumor is positively correlated with bioluminescence intensity $[19,33]$, further investigations into the correlation between intralymphatic tumor volume and bioluminescence intensity are required. Third, although the present study demonstrated an inhibition of tumor growth in the LN, our approach did not eliminate tumor cells completely from the LN. Although the US radiation used in the present study has been shown to improve the delivery of molecules to a solid tumor [19, 34, 35], further work is needed to establish the optimal US conditions for use in metastatic LNs. Fourth, metastatic cells in LNs have the potential to undergo hematogenous metastasis through veins located around the $\mathrm{LN}$ and thus develop a distant metastasis [23]. It will be necessary to determine whether the present methodology is applicable to distant metastasis that occurs by lymphatic spread.

In conclusion, the results of our study suggest that lymphatic administration of drugs via a LN together with sonoporation is a promising method for treating LN metastasis. Lymphatic administration via the SiLN allowed Dox and ALs to reach LVs effectively, leading to the local accumulation of Dox and ALs in the PALN (a model of a LN located outside the lymphadenectomy area). US irradiation of the PALN may trigger cavitation, allowing Dox to extravasate and penetrate into the tumor tissues within the LN. Such action might prevent the spread of metastatic cells further downstream of the LN or to distant organs, providing survival advantages. Moreover, the size of the lymphadenectomy area can be reduced if our novel approach is developed to allow metastatic LNs within the lymphadenectomy area to be completely cleared of tumor.

\section{Supplementary Material}

Additional File 1:

Supplementary Figure S1.

http://www.jcancer.org/v06p1282s1.pdf

Additional File 2:

Supplementary Video SV1.

http://www.jcancer.org/v06p1282s2.avi

\section{Acknowledgments}

This study was supported in part by JSPS KAKENHI grant numbers 15K15690 (SM), 25293382 (SM), 26670856 (MS), 26293425 (MS) and 26242051 (TK).

\section{Competing Interests}

The authors have declared that no competing interest exists.

\section{References}

1. Adams S, Baum RP, Stuckensen T, Bitter K, Hor G. Prospective comparison of 18F-FDG PET with conventional imaging modalities (CT, MRI, US) in lymph node staging of head and neck cancer. European journal of nuclear medicine. 1998; 25: 1255-60.

2. Kelley MC, Hansen N, McMasters KM. Lymphatic mapping and sentinel lymphadenectomy for breast cancer. American journal of surgery. 2004; 188: 49-61.

3. Kodama T, Hatakeyama Y, Kato S, Mori S. Visualization of fluid drainage pathways in lymphatic vessels and lymph nodes using a mouse model to test a lymphatic drug delivery system. Biomedical optics express. 2015; 6: 124-34.

4. Haffty BG, Yang Q, Reiss M, Kearney T, Higgins SA, Weidhaas J, et al. Locoregional relapse and distant metastasis in conservatively managed triple negative early-stage breast cancer. Journal of clinical oncology : official journal of the American Society of Clinical Oncology. 2006; 24: 5652-7.

5. Colnot DR, Nieuwenhuis EJ, van den Brekel MW, Pijpers R, Brakenhoff RH, Snow GB, et al. Head and neck squamous cell carcinoma: US-guided fine-needle aspiration of sentinel lymph nodes for improved staging--initial experience. Radiology. 2001; 218: 289-93.

6. Li L, Mori S, Kodama M, Sakamoto M, Takahashi S, Kodama T. Enhanced sonographic imaging to diagnose lymph node metastasis: importance of blood vessel volume and density. Cancer research. 2013; 73: 2082-92.

7. Primeau AJ, Rendon A, Hedley D, Lilge L, Tannock IF. The distribution of the anticancer drug Doxorubicin in relation to blood vessels in solid tumors. Clinical cancer research : an official journal of the American Association for Cancer Research. 2005; 11: 8782-8.

8. Swartz MA. The physiology of the lymphatic system. Advanced drug delivery reviews. 2001; 50: 3-20.

9. Braun A, Worbs T, Moschovakis GL, Halle S, Hoffmann K, Bolter J, et al. Afferent lymph-derived $\mathrm{T}$ cells and DCs use different chemokine receptor CCR7-dependent routes for entry into the lymph node and intranodal migration. Nature immunology. 2011; 12: 879-87.

10. Dewitte H, Vanderperren K, Haers H, Stock E, Duchateau L, Hesta M, et al. Theranostic mRNA-loaded Microbubbles in the Lymphatics of Dogs: Implications for Drug Delivery. Theranostics. 2015; 5: 97-109.

11. Sever A, Broillet A, Schneider M, Cox K, Jones S, Weeks J, et al. Dynamic visualization of lymphatic channels and sentinel lymph nodes using intradermal microbubbles and contrast-enhanced ultrasound in a swine model and patients with breast cancer. Journal of ultrasound in medicine : official journal of the American Institute of Ultrasound in Medicine. 2010; 29: 1699-704.

12. Goldberg BB, Merton DA, Liu JB, Thakur M, Murphy GF, Needleman L, et al. Sentinel lymph nodes in a swine model with melanoma: contrast-enhanced lymphatic US. Radiology. 2004; 230: 727-34.

13. Shao L, Mori S, Yagishita Y, Okuno T, Hatakeyama Y, Sato T, et al. Lymphatic mapping of mice with systemic lymphoproliferative disorder: usefulness as an inter-lymph node metastasis model of cancer. Journal of immunological methods. 2013; 389: 69-78. 
14. Kato S, Shirai Y, Kanzaki H, Sakamoto M, Mori S, Kodama T. Delivery of Molecules to the Lymph Node via Lymphatic Vessels Using Ultrasound and Nano/Microbubbles. Ultrasound in medicine \& biology. 2015.

15. Hasegawa $\mathrm{H}$, Kohno $\mathrm{M}$, Sasaki $\mathrm{M}$, Inoue $\mathrm{A}$, Ito $\mathrm{MR}$, Terada $\mathrm{M}$, et al. Antagonist of monocyte chemoattractant protein 1 ameliorates the initiation and progression of lupus nephritis and renal vasculitis in MRL/lpr mice. Arthritis and rheumatism. 2003; 48: 2555-66.

16. Sato T, Mori S, Arai Y, Kodama T. The combination of intralymphatic chemotherapy with ultrasound and nano-/microbubbles is efficient in the treatment of experimental tumors in mouse lymph nodes. Ultrasound in medicine \& biology. 2014; 40: 1237-49.

17. Kodama T, Tomita N, Horie S, Sax N, Iwasaki H, Suzuki R, et al. Morphological study of acoustic liposomes using transmission electron microscopy. Journal of electron microscopy. 2010; 59: 187-96.

18. Sax N, Kodama T. Optimization of acoustic liposomes for improved in vitro and in vivo stability. Pharmaceutical research. 2013; 30: 218-24.

19. Watanabe $\mathrm{Y}$, Aoi A, Horie S, Tomita N, Mori S, Morikawa $\mathrm{H}$, et al. Low-intensity ultrasound and microbubbles enhance the antitumor effect of cisplatin. Cancer science. 2008; 99: 2525-31.

20. Tanaka Y, Komori H, Mori S, Soga Y, Tsubaki T, Terada M, et al. Evaluating the role of rheumatoid factors for the development of rheumatoid arthritis in a mouse model with a newly established ELISA system. The Tohoku journal of experimental medicine. 2010; 220: 199-206.

21. Kodama T, Tomita N, Yagishita Y, Horie S, Funamoto K, Hayase T, et al. Volumetric and angiogenic evaluation of antitumor effects with acoustic liposome and high-frequency ultrasound. Cancer research. 2011; 71: 6957-64.

22. Shao L, Ouchi T, Sakamoto M, Mori S, Kodama T. Activation of latent metastases in the lung after resection of a metastatic lymph node in a lymph node metastasis mouse model. Biochemical and biophysical research communications. 2015; 460: 543-8.

23. Shao L, Takeda K, Kato S, Mori S, Kodama T. Communication between lymphatic and venous systems in mice. Journal of immunological methods. 2015.

24. Fan Z, Kumon RE, Park J, Deng CX. Intracellular delivery and calcium transients generated in sonoporation facilitated by microbubbles. Journal of controlled release : official journal of the Controlled Release Society. 2010; 142: 31-9.

25. Qiu Y, Zhang C, Tu J, Zhang D. Microbubble-induced sonoporation involved in ultrasound-mediated DNA transfection in vitro at low acoustic pressures. Journal of biomechanics. 2012; 45: 1339-45.

26. Skyba DM, Price RJ, Linka AZ, Skalak TC, Kaul S. Direct in vivo visualization of intravascular destruction of microbubbles by ultrasound and its local effects on tissue. Circulation. 1998; 98: 290-3.

27. Stieger SM, Caskey CF, Adamson RH, Qin S, Curry FR, Wisner ER, et al. Enhancement of vascular permeability with low-frequency contrast-enhanced ultrasound in the chorioallantoic membrane model. Radiology. 2007; 243: 112-21.

28. Bonnefoi $\mathrm{H}$, Jacot $\mathrm{W}$, Saghatchian $\mathrm{M}$, Moldovan $\mathrm{C}$, Venat-Bouvet $\mathrm{L}$, Zaman $\mathrm{K}$, et al. Neoadjuvant treatment with docetaxel plus lapatinib, trastuzumab, or both followed by an anthracycline-based chemotherapy in HER2-positive breast cancer: results of the randomised phase II EORTC 10054 study. Annals of oncology : official journal of the European Society for Medical Oncology / ESMO. 2015; 26: 325-32.

29. Untch M, Rezai M, Loibl S, Fasching PA, Huober J, Tesch H, et al. Neoadjuvant treatment with trastuzumab in HER2-positive breast cancer: results from the GeparQuattro study. Journal of clinical oncology : official journal of the American Society of Clinical Oncology. 2010; 28: 2024-31.

30. Ah-See ML, Makris A, Taylor NJ, Harrison M, Richman PI, Burcombe RJ, et al. Early changes in functional dynamic magnetic resonance imaging predict for pathologic response to neoadjuvant chemotherapy in primary breast cancer. Clinical cancer research : an official journal of the American Association for Cancer Research. 2008; 14: 6580-9.

31. Sadeghi-Naini A, Papanicolau N, Falou O, Zubovits J, Dent R, Verma S, et al. Quantitative ultrasound evaluation of tumor cell death response in locally advanced breast cancer patients receiving chemotherapy. Clinical cancer research : an official journal of the American Association for Cancer Research. 2013; 19: 2163-74.

32. Hayashi $\mathrm{K}$, Jiang $\mathrm{P}$, Yamauchi $\mathrm{K}$, Yamamoto $\mathrm{N}$, Tsuchiya $\mathrm{H}$, Tomita $\mathrm{K}$, et al. Real-time imaging of tumor-cell shedding and trafficking in lymphatic channels. Cancer research. 2007; 67: 8223-8.

33. Choy G, O'Connor S, Diehn FE, Costouros N, Alexander HR, Choyke P, et al. Comparison of noninvasive fluorescent and bioluminescent small animal optical imaging. BioTechniques. 2003; 35: 1022-6, 8-30.

34. Horie S, Watanabe Y, Ono M, Mori S, Kodama T. Evaluation of antitumor effects following tumor necrosis factor-alpha gene delivery using nanobubbles and ultrasound. Cancer science. 2011; 102: 2082-9.

35. Horie S, Watanabe Y, Chen R, Mori S, Matsumura Y, Kodama T. Development of localized gene delivery using a dual-intensity ultrasound system in the bladder. Ultrasound in medicine \& biology. 2010; 36: 1867-75. 